\title{
Substantial increase in acceleration potential of pyroelectric crystals
}

\author{
W. Tornow, ${ }^{1, a)}$ S. M. Lynam, ${ }^{1, b)}$ and S. M. Shafroth ${ }^{2}$ \\ ${ }^{1}$ Department of Physics, Duke University, Durham, North Carolina 27708, USA and Triangle Universities \\ Nuclear Laboratory, Durham, North Carolina 27708-0308, USA \\ ${ }^{2}$ Department of Physics and Astronomy, University of North Carolina at Chapel Hill, North Carolina \\ 27715, USA
}

(Received 5 January 2010; accepted 17 January 2010; published online 16 March 2010)

\begin{abstract}
We report on a substantial increase in the acceleration potential achieved with a $\mathrm{LiTaO}_{3}$ pyroelectric crystal. With a single $2.5 \mathrm{~cm}$ diameter and $2.5 \mathrm{~cm}$ long z-cut crystal without electric field-enhancing nanotip we produced positive ion beams with maximal energies between 300 and $310 \mathrm{keV}$ during the cooling phase when the crystal was exposed to $5 \mathrm{mTorr}$ of deuterium gas. These values are about a factor of 2 larger than previously obtained with single pyroelectric crystals. (C) 2010 American Institute of Physics. [doi:10.1063/1.3309841]
\end{abstract}

\section{INTRODUCTION}

During the past five years pyroelectric $\mathrm{LiTaO}_{3}$ crystals received quite some attention. It began with the work of the UCLA (Ref. 1) and $\mathrm{RPI}^{2,3}$ groups which reported the production of neutrons using pyroelectric crystals operated in deuterium gas of a few mTorr pressure. The highest positive potential reported by the UCLA group was $115 \mathrm{keV}$. In a double crystal arrangement the RPI group has reported potentials up to $215 \mathrm{keV}$, and recently published a maximum value of about $250 \mathrm{keV}^{4}$ This value corresponds to a single crystal potential of about $125 \mathrm{keV}$. The LLNL (Ref. 5) group observed potentials up to $80 \mathrm{keV}$. In Ref. 6 a maximum positive potential of $165 \mathrm{keV}$ was obtained. In the earlier work of Brownridge et $\mathrm{al}^{7}$ maximal potentials of $113 \mathrm{keV}$ were reported for the case of $\mathrm{LiNbO}_{3}$ crystals.

Considering the pyroelectric crystal and the electron/ion collecting plate (positioned perpendicular to the $\mathrm{z}$ axis of the crystal) as two parallel plate capacitors connected in series, one finds that the acceleration potential is (in very good approximation) proportional to the pyroelectric coefficient $\gamma$, the change in temperature $\Delta \mathrm{T}$, and the length $\mathrm{d}$ of the crystal, and inversely proportional to the product of the dielectric permittivities of free space and of the crystal material. ${ }^{3}$ Counterintuitively, the distance between the face of the crystal and the electron/ion collecting plate does not enter.

Typically, the crystals used in Refs. 1-5 had radii between 1.2 and $1.5 \mathrm{~cm}$ and a length of $1.0 \mathrm{~cm}$. Therefore, it may not be too surprising that the maximal potential achieved was about the same $(80-125 \mathrm{keV})$. On the other hand, in the work of Tornow et al. ${ }^{6}$ longer crystals $(2.5 \mathrm{~cm})$ were used, which may account for the larger potential found in Ref. 6. One of the reasons for not using too long crystals is simply the fact that larger mass crystals are more difficult to heat or cool uniformly at the optimum rate. In addition, a too large temperature gradient across the crystal can cause the crystal to shatter.

\footnotetext{
${ }^{\text {a) }}$ Author to whom correspondence should be addressed. Electronic mail: tornow@tunl.duke.edu.

${ }^{b}$ REU student at TUNL, Summer 2008, from University of Wisconsin at Stevens Point.
}

It is interesting to note that there is a subtle difference between the grounding arrangements used in Refs. 1-5 and in Ref. 6 which may partly be responsible for the higher potential observed in Ref. 6. In the former experimental setups the $\mathrm{z}$ face of the crystal facing the heating/cooling source was grounded, while in the latter it was floating. In the following we report on our improved procedures which resulted in positive ion energies approaching $310 \mathrm{keV}$.

\section{EXPERIMENTAL SETUP AND PROCEDURE}

Figure 1 shows our experimental setup. It consists of a cylindrical vacuum chamber made of aluminum. The bottom flange, also made of aluminum, contains holes and feedthroughs and is connected to a turbomolecular pump and a needle valve which in turn is attached to a gas-filling station. The lid of the cylindrical chamber is made of plastic. At the center of the lid a Si surface barrier detector is mounted which faces the $\mathrm{z}^{+}$face of the crystal. An off-axis feedthrough supports a rotatable disk made of aluminum.

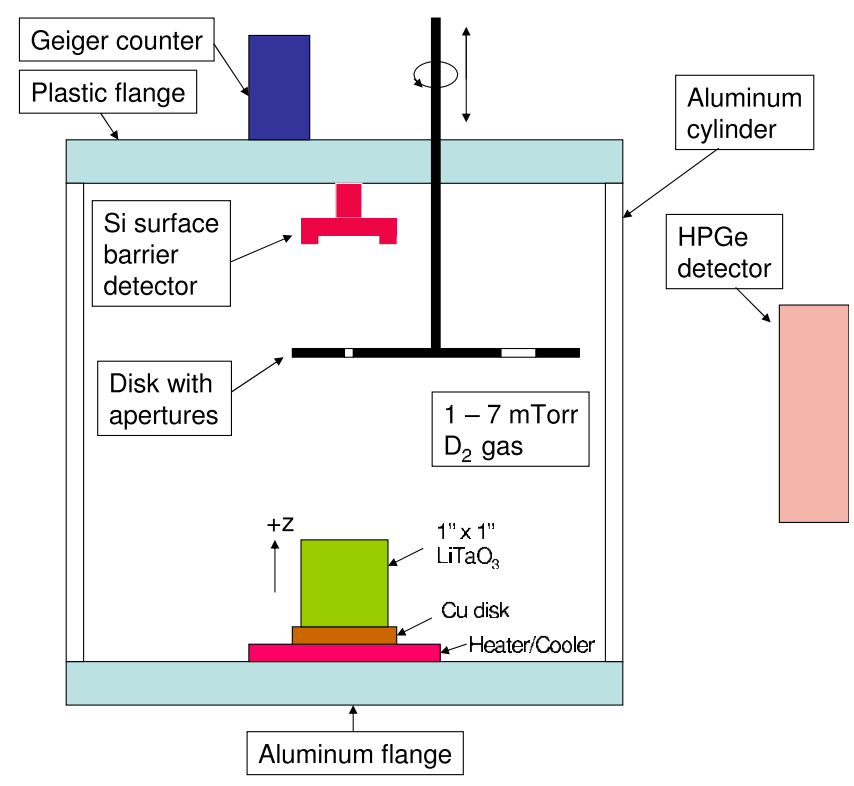

FIG. 1. (Color online) Experimental setup. 
This disk contains apertures of different diameters and can be moved toward or away from the crystal. It not only protects the $\mathrm{Si}$ surface detector from excessive electron and positive ion bombardment, but also serves as charge (current) collector. For most of our experiments the $0.38 \mathrm{~mm}$ diameter aperture of the rotatable disk was positioned above the crystal.

The $2.5 \mathrm{~cm}$ diameter and $2.5 \mathrm{~cm}$ high z-cut $\mathrm{LiTaO}_{3}$ crystal was centered on a $3 \mathrm{~cm}$ diameter and $0.25 \mathrm{~cm}$ thick copper disk which was placed on a heater/cooler device. The latter was in thermal contact with the bottom flange of the vacuum chamber. Note that the $\mathrm{z}^{-}$face of the crystal is not grounded. A thermocouple was attached to the heater/cooler device to monitor its temperature.

A LABVIEW run program was used to adjust the current sent to the heating/cooling device in order to control the heating/cooling rate of the crystal. In addition, the temperature of the crystal, the electron current (during heating), and positive ion current (during cooling) were recorded automatically. Finally, the reading of an end-window Geiger counter placed on top of the chamber to monitor the $\mathrm{x}$-ray radiation produced during heating and cooling of the crystal was recorded automatically. After amplification, the pulse height signals from the $\mathrm{Si}$ detector were digitized and recorded using a multichannel analyzer. In addition to the Si detector, we occasionally used a high-purity germanium (HPGe) detector positioned outside of the vacuum chamber to record the $\mathrm{x}$-ray spectrum during the heating and cooling phase of the crystal.

Once the potential of the pyroelectric crystal is large enough, molecules or atoms approaching the crystal are field ionized. The electrons and positive ions are accelerated by the electric field surrounding the crystal. The resulting electron or positive ion beams are well focused.

The parameters varied were (i) heating rate, (ii) cooling rate, (iii) deuterium pressure, and (iv) distance of the rotating disk from the crystal. Here we report on measurements obtained with a $4.0 \mathrm{~cm}$ distance of the disk from the crystal. Larger and smaller distances resulted in either reduced acceleration potential or enhanced number of discharges. Discharges result when the local electric field exceeds the breakdown potential of the ionized gas. The heating rate was tuned to not cause a discharge before the reading of the thermocouple has reached $130{ }^{\circ} \mathrm{C}$. Heating rates of larger than $20{ }^{\circ} \mathrm{C}$ per minute caused premature discharges and smaller heating rates resulted in less electron current and smaller electron energy. Typically we used a heating rate of $3.5^{\circ} \mathrm{C}$ per minute. Once the temperature reached $70^{\circ} \mathrm{C}$ the electron current exceeded $-0.5 \mathrm{nA}$ and the count rate in our Si detector became so large that a reliable electron energy determination was not possible anymore. By that time the electron energy was typically $200 \mathrm{keV}$, as shown in Fig. 2. Here, the first peak represents a measure of the electron energy. The second peak is part of the multipeak electron energy spectrum typically obtained with pyroelectric crystals. ${ }^{7}$ Most of the counts seen in Fig. 2 at low energies and between the peaks are due to Bremsstrahlung $\mathrm{x}$ rays. Occasionally, we employed the HPGe detector shown in Fig. 1. This detector records the $\mathrm{x}$-ray spectrum generated by the electrons hitting

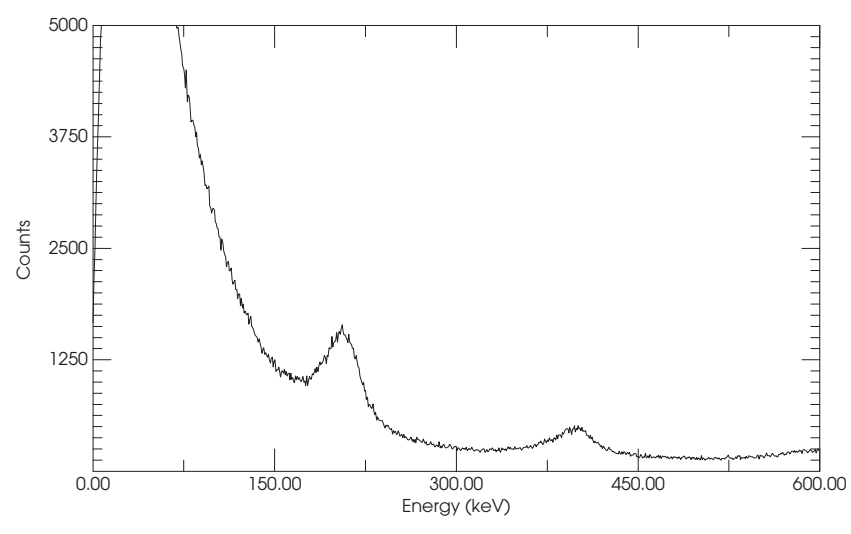

FIG. 2. Electron energy spectrum at high energy.

the disk, the HPGe detector, and the inner walls of the chamber. To reduce the rate in the HPGe detector, we placed a 3.5 $\mathrm{mm}$ thick absorber made of lead in front of this detector.

The heating of our crystal took place at our base vacuum of $<0.4$ mTorr. During the heating phase of the $2.5 \mathrm{~cm}$ tall crystal its actual temperature lags behind the thermocouple reading and, in addition, the $\mathrm{z}^{-}$base is at higher temperature than the top of the crystal. Nevertheless, we did not wait for the crystal temperature to equalize. Once the temperature of the thermocouple read $130{ }^{\circ} \mathrm{C}$, we waited for about $5 \mathrm{~min}$ and, if a discharge did not occur naturally during this time period, we forced a discharge by either adding abruptly deuterium gas or by abruptly turning the rotating disk mounted above the crystal. After a discharge the $\mathrm{z}^{+}$surface of the crystal is neutralized. Of course, the disadvantage of forced discharges at high potentials was the associated increase in the number of cases where our electronic hardware used to control and monitor the crystal functions became disabled. The reason for forcing a discharge at $130{ }^{\circ} \mathrm{C}$ is simply to speed up the neutralization process of the negative polarization charge bound to the top surface of the $\mathrm{z}^{+}$-cut crystal during the heating phase. Because our goal was to reach high positive potentials during the cooling phase of the crystal, any remaining negative polarization charge tends to reduce the achievable positive charge and, therefore, limits the obtainable positive potential. Typically, for our crystal $1-2 \mathrm{~h}$ is needed for the negative polarization charge to neutralize naturally. During this long time our entire vacuum chamber heats up considerably, making it impossible to subsequently cool the crystal at the desired rate to $0{ }^{\circ} \mathrm{C}$.

Right after the desired discharge occurred we selected the deuterium pressure of interest by adjusting the needle valve referred to above. Subsequently we started the cooling process of the crystal. It turned out that the highest positive potential found in the present work was obtained at a cooling rate of about $13{ }^{\circ} \mathrm{C}$ per minute. Once the thermocouple reading reached $0{ }^{\circ} \mathrm{C}$, the positive ion current and the positive ion energy were far from their maximum values yet. In fact, at this time our Si detector still recorded multipeaked electron spectra, while the current reading (obtained from the rotating disk) was already positive, but below $0.2 \mathrm{nA}$. The electric cooler was programmed to keep the thermocouple reading at $0{ }^{\circ} \mathrm{C}$. About 15 min after the thermocouple reading reached $0{ }^{\circ} \mathrm{C}$ the ion energy reached its maximum value 
before discharging. Typically, 2-3 min before the discharge the rate of ion energy increase slowed down considerably (to about $5 \mathrm{keV}$ per minute) and sometimes leveled off, but potentials above $280 \mathrm{kV}$ could not be maintained for more than about $5 \mathrm{~min}$ before a discharge occurred. It is interesting to point out that the ion current and ion energy did not peak at the same time. The ion current reached its maximum value about 1-2 min earlier than the ion energy, which was in phase with the radiation reading of the Geiger counter placed on top of the chamber. At ion energies above $250 \mathrm{keV}$ we occasionally recorded radiation levels higher than 200 $\mathrm{mrem} / \mathrm{h}$, when the Geiger counter was placed on top of our chamber.

Cool-down times between 10 and 20 min provided about the same maximal ion energies, and in most cases the associated potentials could be maintained over a somewhat longer period of time with the 20 min cool-down time, although ultimately we always experienced a discharge. Only for cool-down times longer than $30 \mathrm{~min}$, which resulted in maximal ion energies of about $250 \mathrm{keV}$, discharges did not usually occur.

\section{RESULTS}

The upper panel of Fig. 3 shows the dependence of maximal positive ion current on the deuterium gas pressure in our chamber. Clearly, the ion current decreases rapidly with increasing pressure, especially at pressures below 2 mTorr. A decrease is expected from simply considering the mean free path of deuterium ions. Furthermore, the steep decrease observed in the present work seems to support the conjecture of Gillich et al. ${ }^{8}$ that the ion beams generated with pyroelectric crystals consist mainly of $\mathrm{D}_{2}^{+}$ions and not $\mathrm{D}^{+}$ ions. ${ }^{8}$ At 1 and 6 mTorr the mean-free path of $\mathrm{D}_{2}^{+}$ions is about 15 and $2.5 \mathrm{~cm}$, respectively, compared to the $4 \mathrm{~cm}$ distance between the top of the crystal and the current collecting disk. The bottom panel of Fig. 3 gives our result for the maximum positive ion energy obtained in the present work as a function of deuterium pressure. The ion energy increases from about $250 \mathrm{keV}$ at base vacuum to about 300 $\mathrm{keV}$ at deuterium pressures above 5 mTorr. With our Si surface barrier detector we routinely measured ion energies between 300 and $310 \mathrm{keV}$ just before the crystal discharged. Our energy calibration is based on measurements with a ${ }^{14} \mathrm{C}$ electron source (end point energy of $154 \mathrm{keV}$ ) and a ${ }^{241} \mathrm{Am}$ alpha-particle source $(5.486 \mathrm{MeV})$. In the latter case calibrated pulse-height attenuators were used to cover the pulseheight range of interest. The uncertainty in our knowledge of the absolute energy scale is about $3 \mathrm{keV}$.

Figure 4 shows a pulse-height spectrum with maximal ion energy of $306 \mathrm{keV}$. The accumulation time was about 15 s. Here, the $0.38 \mathrm{~mm}$ diameter aperture of the disk was positioned in front of the Si detector. Most of the counts in this spectrum seen at lower energies are not due to positive ions, but due to $\mathrm{x}$ rays recorded by the Si detector. This can be proven by simply rotating the aperture a small amount so that the ion beam is absorbed in the aluminum disk. This
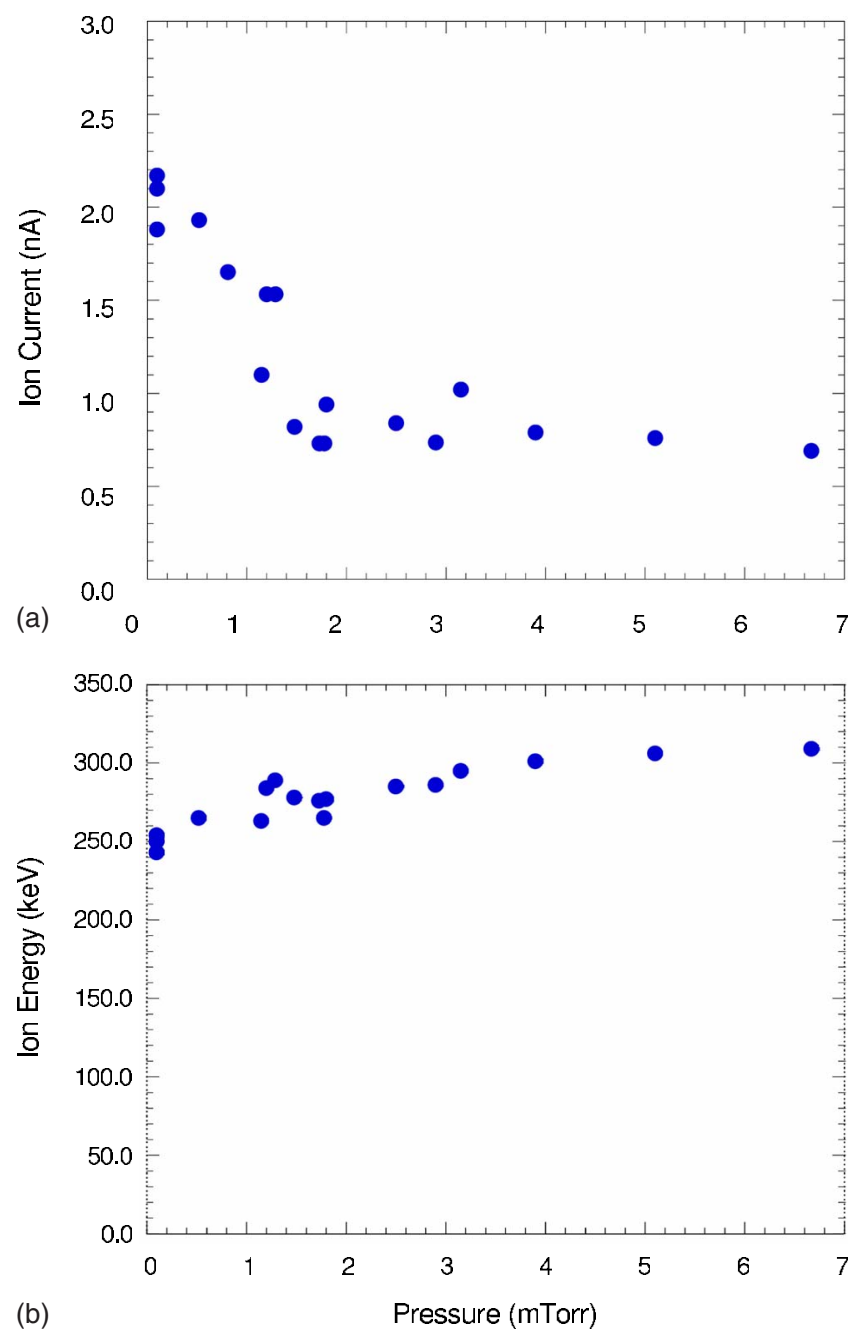

FIG. 3. (Color online) Top panel: maximal ion current vs deuterium pressure. Bottom panel: maximal positive ion energy vs deuterium pressure.

misalignment results in a pulse-height spectrum similar to the one shown in Fig. 4, but without a peak at the highest energy.

An independent verification of the high positive ion energies measured in the present work was obtained with our HPGe detector. The two panels of Fig. 5 show the highenergy part of an x-ray spectrum recorded with the $3.5 \mathrm{~mm}$

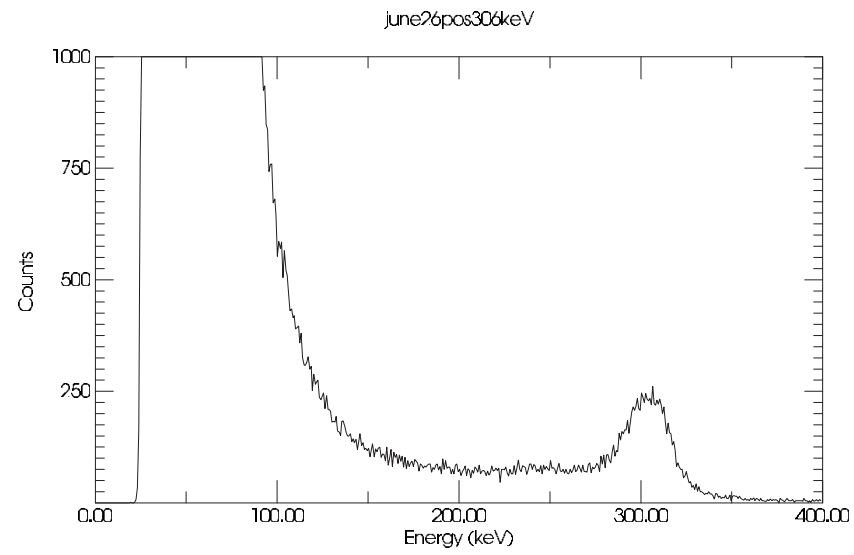

FIG. 4. Pulse-height spectrum obtained with Si surface barrier detector with maximum positive ion energy of $306 \mathrm{keV}$. 

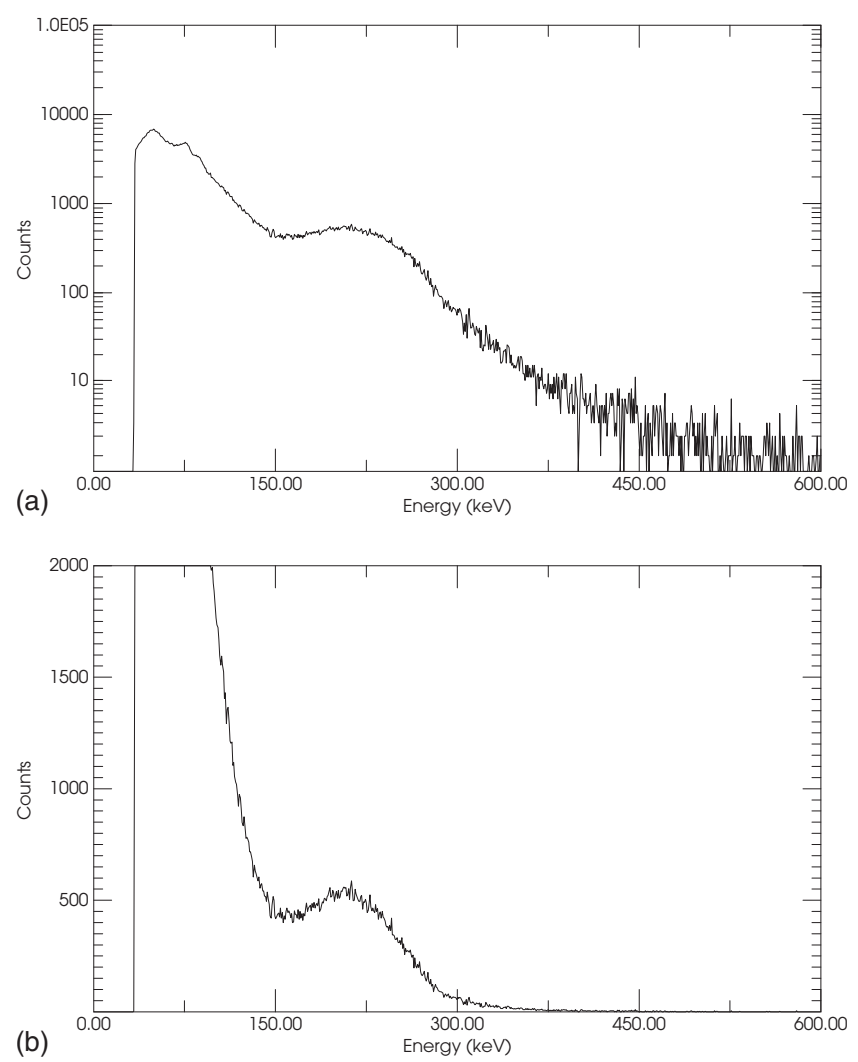

FIG. 5. Panels (a) and (b): High-energy part of x-ray pulse-height distribution obtained with HPGe detector shown with logarithmic and linear vertical scales, respectively. The associated positive ion spectrum obtained with the Si surface barrier detector is shown in Fig. 4.

thick $\mathrm{Pb}$ absorber placed in front of the $25 \%$ efficient HPGe detector, which was at a distance of $40 \mathrm{~cm}$ from the crystal. Panel (a) shows the spectrum using a logarithmic scale, while panel (b) shows the same spectrum on a linear vertical scale. Clearly, using the logarithmic scale, extrapolation to the end point results in positive ion energies well above 300 $\mathrm{keV}$. However, this extrapolation is misleading due to pile-up in the associated amplifiers caused by the high rate. From panel (b) a more reliable end point energy can be extracted, which lies definitely near $300 \mathrm{keV}$.

Before concluding, we would like to point out that we did not try to optimize the conditions for obtaining the highest electron energy. The maximal electron energy found in the present work and measured with both the Si surface barrier detector and the HPGe detector was $225 \mathrm{keV}$. The associated Geiger counter readings were about $75 \mathrm{mrem} / \mathrm{h}$. However, we feel that we were not too far off from the optimal conditions. This observation confirms our earlier finding ${ }^{6}$ that it is apparently easier to obtain high positive ion energies than high electron energies.

\section{SUMMARY}

In summary, with a $2.5 \mathrm{~cm}$ diameter and $2.5 \mathrm{~cm}$ high $\mathrm{LiTaO}_{3}$ pyroelectric crystal we obtained unprecedented high positive potentials between 300 and $310 \mathrm{keV}$. The main difference between our previous approach ${ }^{6}$ which resulted in positive potentials of up to $165 \mathrm{keV}$ and the present work is the use of a metallic (aluminum) chamber with a plastic lid compared to a plastic chamber and a metallic (aluminum) lid used previously. In addition, the present LABVIEW based setup made it possible to fine tune the operating parameters of the crystal, which was not possible in Ref. 6. In contrast with the pyroelectric crystal arrangements of the UCLA and RPI groups, we did not employ an electric field-enhancing nanotip. A nanotip may prevent the crystal from acquiring very high potentials due to the loss of charge, but it should provide larger ion currents. The other major difference between the arrangements of the UCLA and RPI groups and our present setup is the fact that we do not ground the side of the crystal facing the heating/cooling device.

The high positive ion energies achieved in the present work extend the range of potential applications of pyroelectric crystals to a new domain in science and technology. Because the figure-of-merit for producing neutrons via the ${ }^{2} \mathrm{H}(\mathrm{d}, \mathrm{n})^{3} \mathrm{He}$ reactions is $\mathrm{IE}^{3 / 2}$ in the energy region of interest, where $\mathrm{I}$ is the positive $\mathrm{D}_{2}^{+}$current and $\mathrm{E}$ is the associated ion energy, a sizeable increase in $\mathrm{E}$ will result in a substantial increase in neutron yield. In addition, a double crystal arrangement similar to the one employed by the RPI group has the potential of increasing the positive ion energy even more. Furthermore, the ion beams (deuterium, nitrogen, and oxygen), electron beams, and $\mathrm{x}$ rays produced in the present work could replace existing technologies and could extend the applications of those beams to new fields.

\section{ACKNOWLEDGMENTS}

The authors would like to thank B. Carlin, G. Rich, J. Cox, C. Westerfeldt, and A. S. Crowell for their contributions to this work. This work was partially supported by the U.S. Department of Energy, Office of Nuclear Physics under Grant No. DE-FG02-97ER41033.

${ }^{1}$ B. Naranjo, J. K. Gimzewski, and S. Putterman, Nature (London) 434, 1115 (2005).

${ }^{2}$ J. Geuther, Y. Danon, and F. Saglime, Phys. Rev. Lett. 96, 054803 (2006).

${ }^{3}$ J. Geuther and Y. Danon, Appl. Phys. Lett. 90, 174103 (2007).

${ }^{4}$ D. Gillich, Nucl. Instrum. Methods Phys. Res. A A602, 306 (2009).

${ }^{5}$ V. Tang, Rev. Sci. Instrum. 78, 123504 (2007).

${ }^{6}$ W. Tornow, S. M. Shafroth, and J. D. Brownridge, J. Appl. Phys. 104, 034905 (2008)

${ }^{7}$ J. D. Brownridge, S. M. Shafroth, D. Trott, B. Stoner, and W. Hooke, Appl. Phys. Lett. 78, 1158 (2001).

${ }^{8}$ D. Gillich, "Making the renaissance real," Proceedings of the ANS/ENS International Meeting, Washington, D.C., 2007 (unpublished), p. 927. 